\title{
miR-30a-3p inhibits renal cancer cell invasion and metastasis through targeting ATG12
}

\author{
Yonghui Chen", Jiale Zhou", Xiaorong Wu, Jiwei Huang, Wei Chen, Dongming Liu, Jin Zhang, \\ Yiran Huang, Wei Xue \\ Department of Urology, Renji Hospital, School of Medicine, Shanghai Jiaotong University, Shanghai 200127, China \\ Contributions: (I) Conception and design: Y Chen, J Zhou, W Xue; (II) Administrative support: W Xue, Y Huang; (III) Provision of study materials or \\ patients: X Wu, J Huang, W Chen; (IV) Collection and assembly of data: J Zhou, X Wu, Y Chen; (V) Data analysis and interpretation: J Zhou; (VI) \\ Manuscript writing: All authors; (VII) Final approval of manuscript: All authors. \\ "These authors contributed equally to this work. \\ Correspondence to: Yonghui Chen; Wei Xue. Department of Urology, Renji Hospital, School of Medicine, Shanghai Jiaotong University, Shanghai \\ 200127, China. Email: cyh1488@163.com; xuewei@renji.com.
}

\begin{abstract}
Background: Aberrantly expressed microRNAs play important biological functions in the pathogenesis, progression and metastasis of numerous malignancies. Thus, further identification of these non-coding transcriptions is warranted.

Methods: miRNAs expression data of renal cell carcinoma (RCC) as well as the normal renal tissue samples was downloaded from TCGA and GEO database, and was analyzed by both computational and experimental approaches. The bio-functional role of the miRNA was then moreover identified by over-expression and inhibition assays. Beyond that, Western blots, luciferase assays, along with immunohistochemistry (IHC) tests were conducted for further study of the potential mechanisms.

Results: In this study, a novel regulatory miRNA, miR-30a-3p, was identified along with its biological function as well as prognostic value in RCC. Functionally, miR-30a-3p inhibits RCC cell invasion and migration. miR-30a-3p targets autophagy related 12 (ATG12), which we confirmed by luciferase reporter assays and Western blotting.

Conclusions: In conclusion, miR-30a-3p, as a new prognostic marker in RCC, down-regulates RCC cell invasion and migration by targeting ATG12. This consistently improves the overall survival of RCC patients.
\end{abstract}

Keywords: miR-30a-3p; ATG12; renal cell carcinoma (RCC)

Submitted Jun 11, 2019. Accepted for publication Nov 05, 2019.

doi: $10.21037 /$ tau.2019.12.10

View this article at: http://dx.doi.org/10.21037/tau.2019.12.10

\section{Introduction}

Nowadays, renal cell carcinoma (RCC) is recognized as one among the most common urological malignancies worldwide, accounting for barely $2-3 \%$ of all human malignancies, with approximately 65,340 newly diagnosed patients and 14,970 deaths in 2018 in U.S. alone $(1,2)$. Nevertheless, with the rapid growth of image technology, more RCCs can be diagnosed at an early stage, before the presentation of any clinical symptoms. However, nearly $30 \%$ of initially diagnosed RCC patients still suffer from metastatic lesions (3). Previous studies have shown RCCs are not sensitive to either radio- or chemotherapy. Thus, the long-term prognostic outcome of patients with metastatic RCCs remains unfavorable, even after cytoreductive surgery. The first-line therapies for advanced RCCs includes immunotherapies and targeted therapies. However, tolerance against the above therapies would soon develop shortly after the initiation of therapy, leading to the low overall survival (OS) of patients with advanced RCCs (4). At this point, a further study of the mechanism which induces the pathogenesis, progression and metastasis of 
RCC, based on which more effective treating methods can be developed, is urgent for urologists.

MicroRNAs (miRNAs), commonly recognized as a family of small non-coding RNA molecules containing about 22 nucleotides in length, have been reported to negatively regulate gene expression (5). Many studies have indicated miRNAs contribute to several metastatic steps of various human malignancies (6). Furthermore, several miRNAs were reported to participate in RCC invasion and metastasis, such as miR-452-5p, miR-532-5p, and miR$766-3 p(3,7,8)$. A great amount of evidence indicates that miR-30a-3p plays influential roles in tumor suppression in multiple kinds of tumors $(9,10)$. However, its biological function in RCC has not been fully studied.

Autophagy related protein 12 (ATG12) is a human homolog of a yeast protein involved in autophagy, which is a process of cellular component degradation and reutilization. Beyond that, it can also kill cells through an autophagyindependent process (11). In previous studies, AGT12 was found to be down-regulated by oncogenic RAS in colorectum malignancies and prolonged OS (12). However, the correlation between ATG12 and miRNAs in RCC has not been studied.

In this article, we investigated bio-function of miR$30 \mathrm{a}-3 \mathrm{p}$, which is a novel tumor suppressor inhibiting RCC invasion and migration. Furthermore, the downstream target gene of miR-30a-3p, ATG12, was recognized and its biological roles in RCC progression was verified.

\section{Methods}

\section{Tissue samples}

The use of human tissues was authorized by research medical ethics committee of Shanghai Renji Hospital \{Ethics board approval number: Renji Ethics [2017]091\}. RCC samples and paired normal renal tissue samples were obtained from the Department of Urology, Renji Hospital Affiliated to Shanghai Jiaotong University, School of Medicine. The fresh tissues obtained from clinic were preserved in liquid nitrogen for prevention of RNA degradation.

\section{Cell culture}

Both of the human RCC cell lines, A498 and 786-O, as well as the human normal renal cell line HK-2 were originally purchased from Cell Bank of the Chinese Academy of
Sciences (Shanghai, China). All cell lines were cultured as previously described (7).

\section{Cell transfection and vector construction}

Transfection and vector construction was performed as we described previously (7). and after 48 hours of transfection, the cells were harvested for the following experiments.

\section{Wound-bealing and invasion assay}

For the purpose of investigating invasion and migration, transwell and scratch assays were both performed as previously described (7). The two experiments were repeated for more than three times to ensure the similar outcomes.

\section{Luciferase reporter assay}

For the verification of ATG12 as the downstream target gene of miR-30a-3p, we performed luciferase reporter assays. The assay was performed as we described previously (7).

\section{Protein extraction and Western blot analysis}

Protein extraction and Western blot analysis was performed as we described previously.

\section{Immunohistochemistry (IHC)}

IHC was conducted with a primary antibody against ATG12 (1:100, Proteintech, 2011S, Wuhan, China) and a secondary antibody (1:200, HRP, Servicebio, GB23303). IHC and calculating of staining index was performed as we described previously (7). Cells were scored only when the clear tumor cell morphology was observed.

\section{Results}

\section{miR-30a-3p is down-regulated in RCC tissues}

For purpose of understanding the potential mechanism of RCC cell invasion, metastasis, and tolerance of target therapy, thus explore differently expressed miRNAs and their biological roles in RCC, an overlap of several miRNA arrays from the GEO sets (Figure 1A), which compared RCC tissues with normal tissues (GSE109368, GSE73342, and GSE12105), metastatic RCC with non-metastatic RCC 

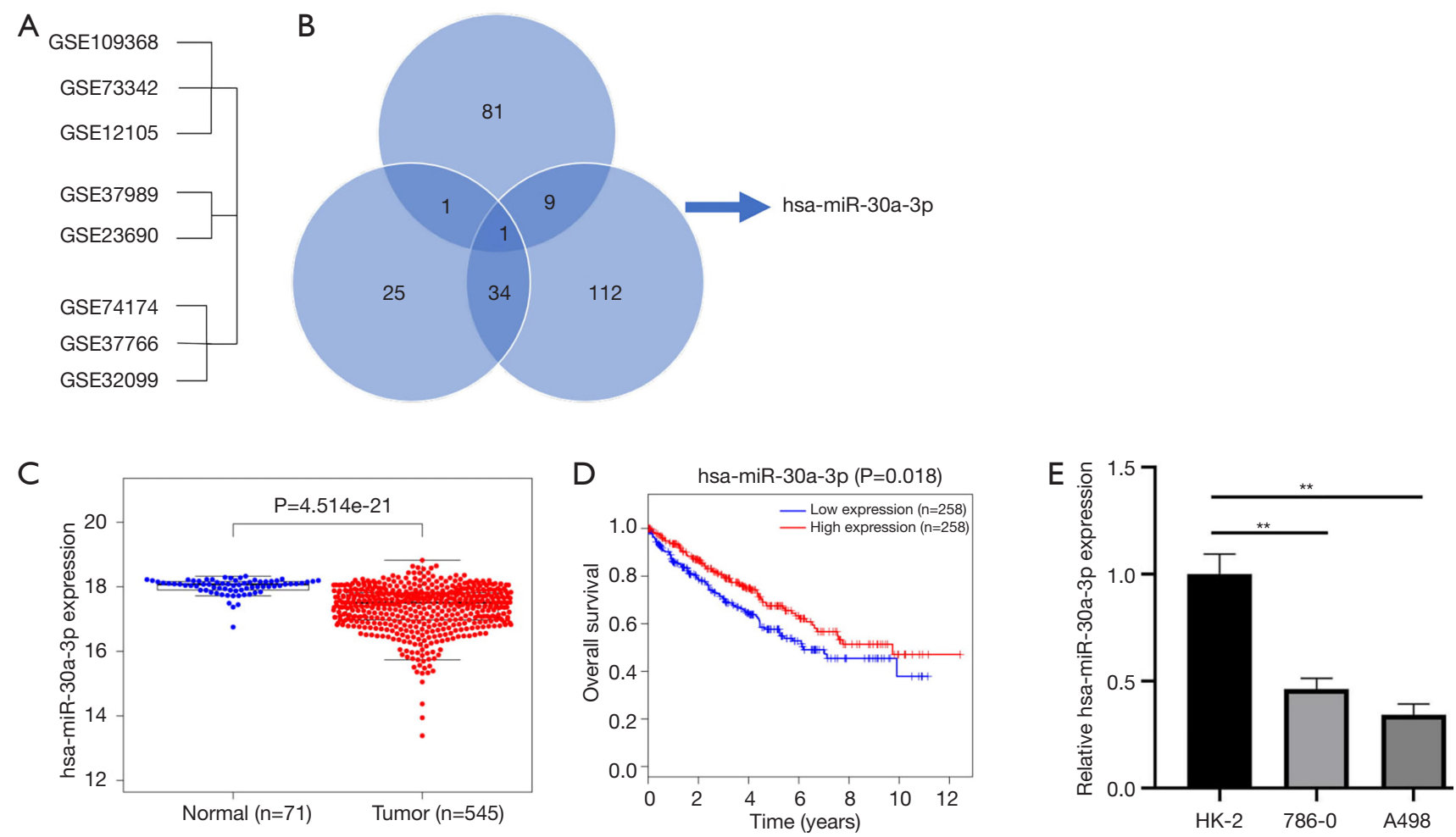

Figure 1 miR-30a-3p is down-regulated in RCC tissues. (A) the flow chart shows the miRNA arrays from the GEO database. (B) The Venn figure shows the overlap of differentially expressed miRNAs from GEO datasets. (C) miR-30a-3p expression in RCC comparing to normal tissues from TCGA database. (D) Kaplan-Meier analysis of the correlation between the expression of miR-30a-3p and the OS of 516 RCC patients from the TCGA RCC database. (E) The expression of miR-30a-3p in cell lines (786-O, A498 and HK-2). **, P<0.01. RCC, renal cell carcinoma; OS, overall survival.

(GSE37989 and GSE23690), and target therapy sensitive with insensitive (GSE74174, GSE37766, and GSE32099) was conducted. It turned out that only miR-30a-3p overlapped in the Venn map (Figure 1B), and it had not been previously reported in RCC before.

To assess the status of miR-30a-3p expression in RCC tissues, data from the TCGA database was analyzed. It was found that miR-30a-3p expression was down-regulated in RCC tissues compared with that in normal renal tissues (17.506 vs. 18.076, $\mathrm{SD}=0.683$ ) (Figure 1C). Furthermore, a Kaplan-Meier curve revealed a positive correlation between miR-30a-30 expression and OS (Figure 1D).

Two cell lines were used for further exploration of different expression levels of the miR, with $\mathrm{HK}-2$ as a contraction. The expression of miR-30a-3p was decreased in RCC cell lines when compared to the normal control (HK-2) (Figure 1E).

\section{miR-30a-3p inhibits RCC migration and invasion in vitro}

For the further investigation of miR-30a-3p in RCC progression, we transfected miR-30a-3p inhibitor or miR-30a-3p NC into A498 and 786-O RCC cells. Transwell invasion assays suggested miR-30a-3p inhibitor transduction in both cell lines turned out to be more invasive when compared with the NC group. On the other hand, knocking-down miR-30a-3p expression (miR-30a$3 p$ inhibitor) enhanced cell migration when compared to control group (miR-30a-3p NC) (Figure 2A,B,C).

Then, we purposed to further study whether miR$30 a-3 p$ can inhibit RCC cell migration and invasion. For this reason, miR-30a-3p mimic or miR-30a-3p NC was transfected into both cell lines, and the transwell invasion assay and migration assay were completed. Compared with the control group, the miR-30a-3p mimic group 

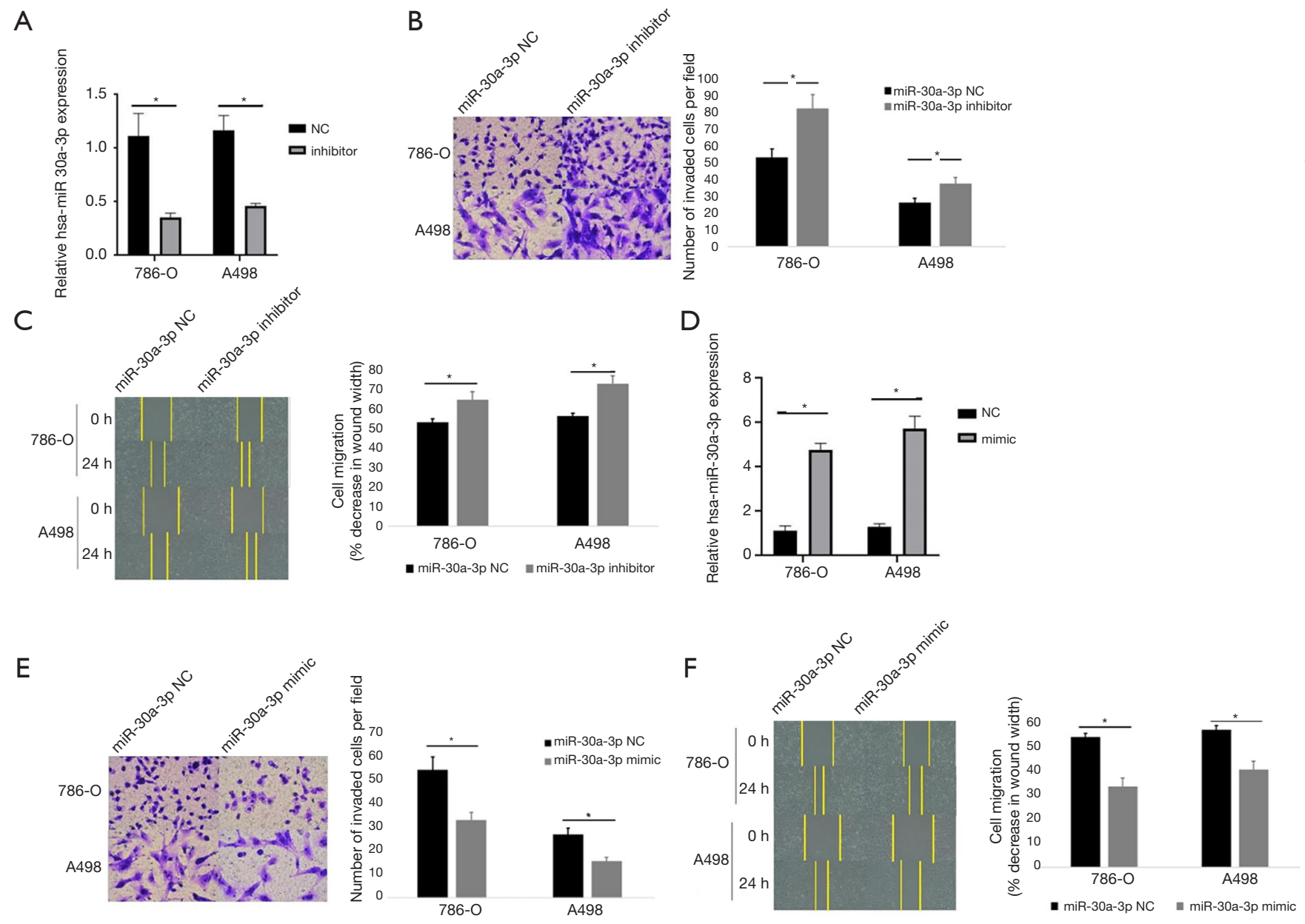

Figure 2 miR-30a-3p inhibits RCC cell invasion and migration in vitro. (A) qRT-PCR assays for miR-30a-3p inhibitors in 786-O and A498 RCC cells versus miR-30a-3p NC cells, respectively. (B) Result of Transwell assay conducted by transfection of miR-30a-3p inhibitors in 786-O and A498 RCC cells versus miR-30a-3p NC cells (crystal violet staining, 200x). (C) Result of wound-healing assays conducted by transfection of miR30a-3p inhibitors in A498 and 786-O RCC cells versus miR-30a-3p NC cells. (D) qRT-PCR assays for miR-30a-3p mimics in 786-O and A498 RCC cells versus miR-30a-3p NC cells, respectively. (E) Result of Transwell assay conducted by transfection of miR-30a-3p mimics in 786-O and A498 RCC cells versus miR-30a-3p NC cells (crystal violet staining, 200x). (F) Result of wound-healing assays conducted by transfection of miR30a-3p mimics in A498 and 786-O RCC cells versus miR-30a-3p NC cells. *, $\mathrm{P}<0.05$. RCC, renal cell carcinoma.

could effectively inhibit the invasion of RCC cells in the transwell assay. Beyond that, in the wounding healing assay, the decrease in the wound width was lower in miR-30a-3p group, which demonstrates miR-30a-3p can inhibit RCC cell migration (Figure 2D,E,F).

Taken together, the data above revealed that miR-30a- $3 \mathrm{p}$ possibly functions as a tumor suppressive miRNA in RCC.

\section{miR-30a-3p targets ATG12 by binding its 3'UTR}

For further studies of miR-30a-3p regarding its roles in the development and progression of RCC, we analyzed the signaling pathway and target genes of miR-30a-3p. Analysis of the potential signaling pathways of miR-30a-3p was done based on the KEGG database (Figure $3 A$ ). According to the analysis, we found that the FOXO signaling pathway was the most differently expressed pathway. Then, we searched for the potential genes encompassed in this signaling pathway, and 79 genes were involved. In addition, we explored the possible target genes of miR-30a-3p through computational approach. Notably, the overlap in these two groups of genes was ATG12 (Figure 3B). Next, we used 
A

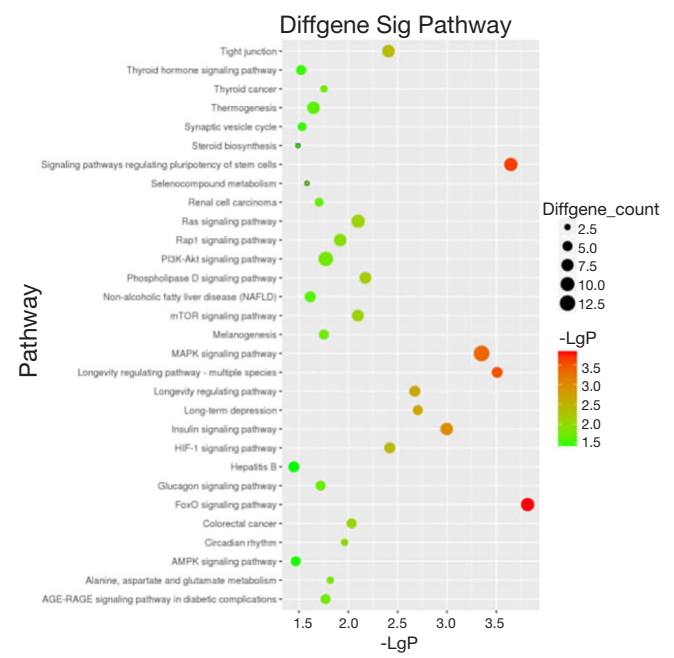

B

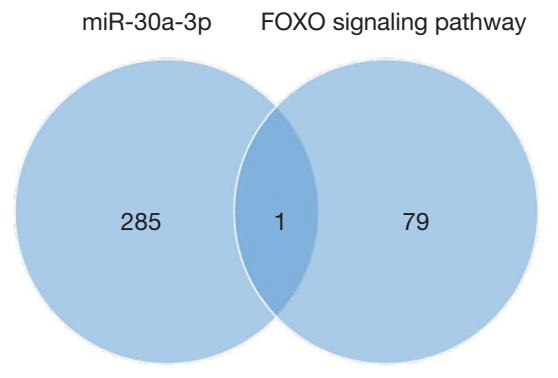

C

\begin{tabular}{|c|c|}
\hline Position 119-125 of ATG12 3' UTR WtA & 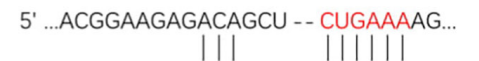 \\
\hline hsa-miR-30a-3p & CGACGUUUGUAGGCUGACUUUC \\
\hline ATG12 3' UTR mutA & $5^{\prime} \ldots$ ACGGAAGAGACAGCU $\ldots-\cdots$ - AG... \\
\hline Position 388-394 of ATG12 3' UTR WtB & 5' ...UCAGUUCUCAAAAUGACUGAAAU... \\
\hline hsa-miR-30a-3p & CGUCGUUUGUAGACUGACUUUC \\
\hline ATG12 3' UTR mutB & 5' ...UCAGUUCUCAAAAUG--- \\
\hline Position $649-655$ of ATG12 3' UTR WtC & $\begin{array}{r}5^{\prime} \quad \ldots \text { AAGCACAUAGGACAGACUGAAAG... } \\
\|\|\|\|\|\|\end{array}$ \\
\hline hsa-miR-30a-3p & CGACGUUUGUAGGCUGACUUUC \\
\hline 3' UTR mutC & GCACAL \\
\hline
\end{tabular}

D

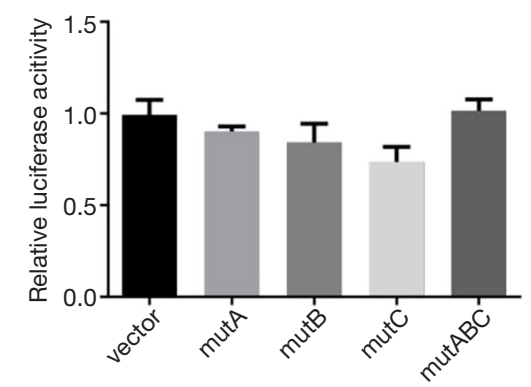

$\mathrm{E}$
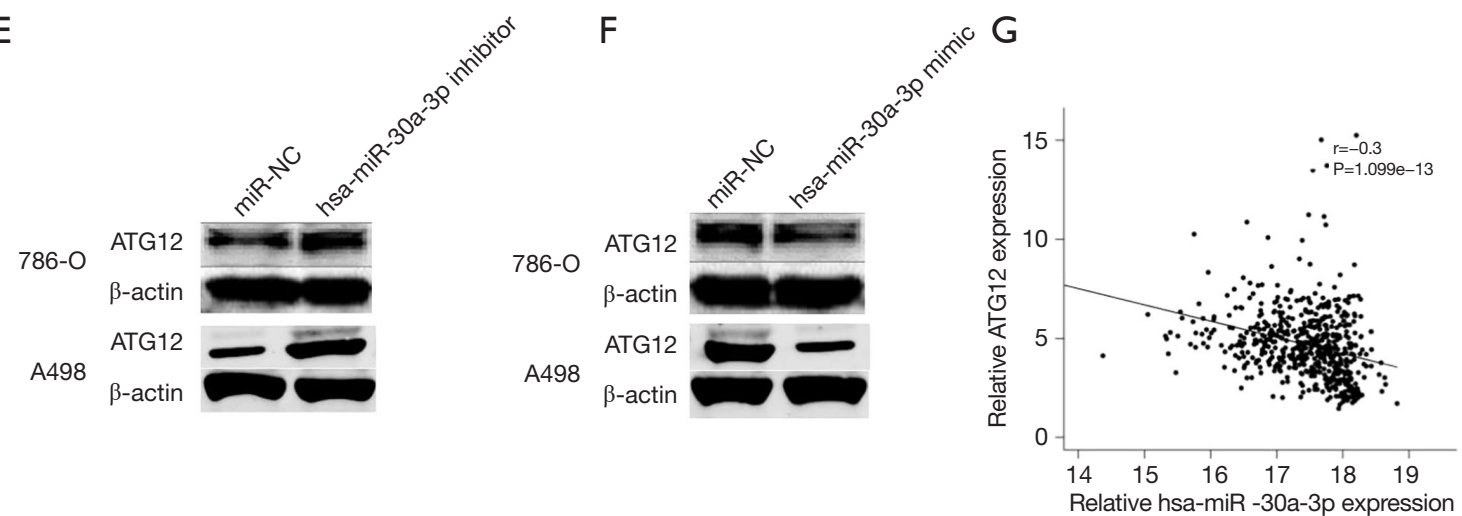

Figure 3 miR-30a-3p targets ATG12 by binding its 3'UTR. (A) Analysis of the potential signaling pathways of miR-30a-3p according to the KEGG database. (B) Venn figure indicated ATG12 was the only overlap of genes involved in FOXO signaling pathway and potential target genes of miR-30a-3p. (C) Bioinformatic analysis reveals the potential ATG12-binding sites in miR-30a-3p. (D) Luciferase reporter assays of ATG12 in 786-O cells. (E,F) Western blot of ATG12 protein levels in miR-30a-3p compared to miR-NC. (E), miR-30a-3p inhibitor compared to miR-NC inhibitor; and (F) miR-30a-3p mimic compared to miR-NC mimic. (G) Spearman's correlation analysis of miR-30a$3 p$ and ATG12 expression in RCC tissues from TCGA database. RCC, renal cell carcinoma. 
the bioinformatics analysis according to miRNA database (TargetScan), and three putative binding sites for miR$30 \mathrm{a}-3 \mathrm{p}$ were found in the 3 ' untranslated region (UTR) of ATG12 (Figure 3C). Therefore, a luciferase reporter assay was accomplished to check whether ATG12 plays a biological function as a target gene of miR-30a-3p, and the results confirmed our hypothesis (Figure $3 D$ ). In addition, Western blotting analysis was performed for the detection of protein levels of ATG12. When the miR-30a-3p mimic was introduced into the 786-O cell line, a decrease in ATG12 levels was observed. In contrast, the level of ATG12 increased when miR-30a-3p was inhibited (Figure 3E,F). The data from the TCGA database also indicated there exists a negative relationship between the expression of ATG12 and the expression of miR-30a-3p in RCC tissues (Figure 3G).

\section{Low expression of ATG12 indicates better RCC prognosis}

To confirm the correlation between ATG12 and the prognosis of RCC, the TCGA RCC database was used, and the result indicated higher expression of ATG12 in RCC tissues compared with normal renal tissues (Figure 4A). Furthermore, the expression of ATG12 was higher in RCC tissues with a higher $\mathrm{T}$ stage (stage III-IV) versus lower stage (stage I-II) (Figure 4B). Next, we performed a KaplanMeier analysis of the correlation between the expression of ATG12 and the OS of 877 RCC patients according to a computational prediction (GEPIA), and the result indicated patients with lower levels of ATG12 benefits from a better OS (Figure $4 C$ ). In addition, the relationship between the expression of ATG12 and disease-free survival was predicted by the same method and showed a similar result (Figure 4D). IHC staining of the ATG12 gene in RCC tissues and normal tissues also showed that gene was up-regulated in RCC tissues (staining index $=8$ ), rather than in normal renal tissues (staining index $=1$ ) (Figure 4E).

Taken together, the downregulation of ATG12 correlated with a better prognosis of RCC.

\section{Discussion}

In the current study, we first revealed miR-30a-3p as a potential tumor suppressing miR of RCC metastasis and is statistically correlated with a better prognosis. The expression level of miR-30a-3p was obviously low in both RCC samples and cell lines, when compared to normal tissues and HK-2 cell line. In addition, ATG12, an autophagy promoter, turned out to be the downstream target gene of miR-30a-3p, and at the same time, is a prognostic marker indicating a poor oncologic outcome of RCC. Consistently, miR-30a-3p inhibits cell migration and invasion in vitro and inhibits RCC metastasis by targeting ATG12. Among the above evidences, it can thus be suggested that miR-30a-3p acts as a tumor suppressor and a metastasis-inhibiting microRNA in RCC.

In reviewing the literature, several reports showed that miR-30a-3p was a tumor suppressor in multiple kinds of malignancies. For example, the low expression of miR$30 a-3 p$ is directly related to poor OS and recurrence free survival in triple-negative breast cancer (9). In lung adenocarcinoma, miR-30a-3p has already shown its value as a prognostic predicting factor (13). In another study, miR-30a-3p has been demonstrated to have the biological function in inhibiting proliferation, invasion, and metastasis in hepatocellular carcinoma (HCC) (14). However, the biological role of miR-30a-3p in the progression and metastasis of RCC remained unclear. Therefore, we conducted our study, and identified its biological function in RCC, as an anticarcinogenesis miRNA.

For the purpose of identifying targets of miR-30a-3p in RCC, several target-predicting online software and datasets were used. And we found ATG12 is a potential target gene of miR-30a-3p. ATG12 is a key protein involved in the elongation and maturation of autophagy vesicles (11). One study showed ATG12 plays as a pro-autophagic protein in breast cancer and is involved in the regulation of epirubicin sensitivity (15). In colorectal cancer, the inhibition of ATG12-mediated autophagy is correlated with the improvement of radiosensitivity (12). In another study, ATG12 was reported to be a target gene of miR-378, which enhances migration and invasion in cervical cancer (16). In our study, ATG12 was proved to be highly expressed in RCC cells and had a negative relationship with the prognosis of RCC.

Unlike the favorable prognosis of localized RCC, metastatic RCC is accompanied by rather poor oncological outcomes. Thus, further study of the biological mechanism behind the invasion and migration of RCC cells is urgent and important. In conclusion, this finding may help us to better understand the role of $\mathrm{miR}-30 \mathrm{a}-3 \mathrm{p}$ in progression and metastasis of RCC by targeting ATG12. The result may have important implications for developing prognostic model or further therapeutic methods. 
A

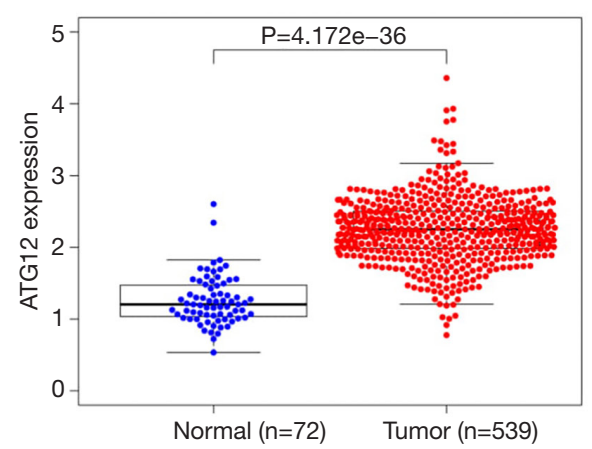

C

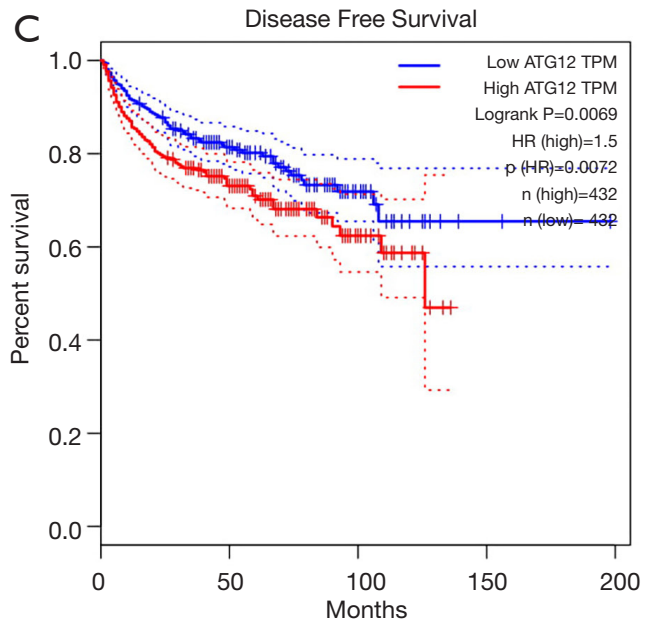

$\mathrm{E}$

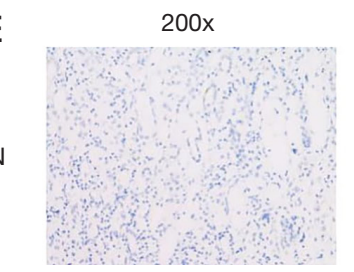

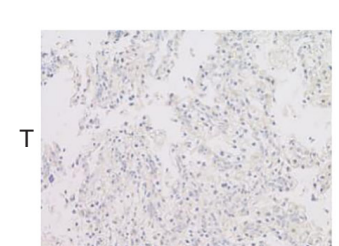

B

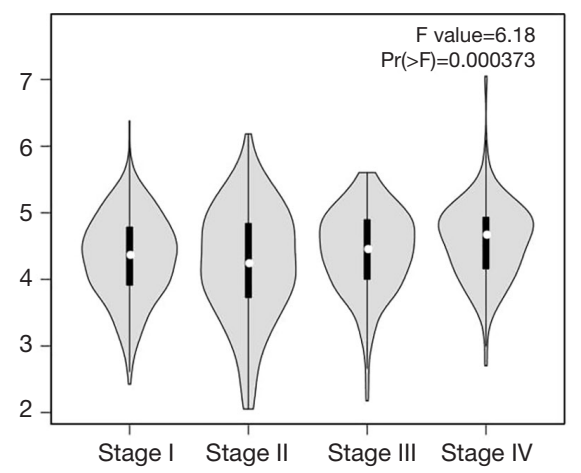

D

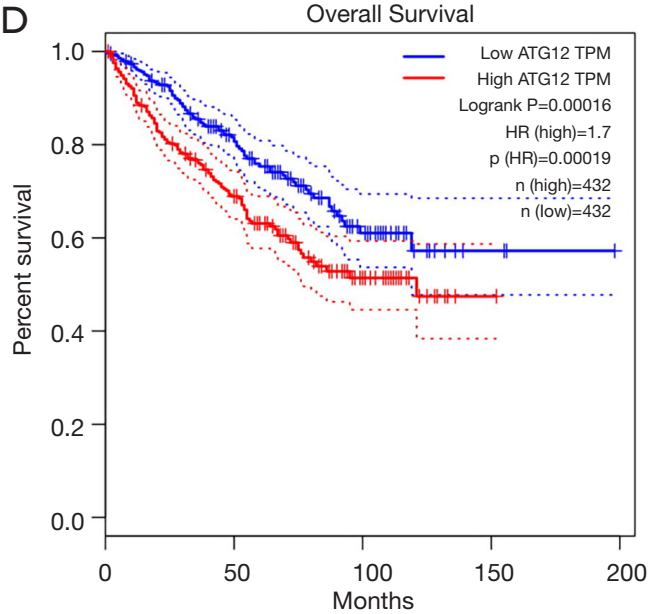

Figure 4 Low expression of ATG12 indicates better RCC prognosis. (A) The expression of ATG12 in RCC and normal renal tissue samples from the TCGA RCC database. (B) ATG12 expression in different RCC TNM stages according to the TCGA RCC database. (C) ATG12 expression and disease-free survival (DFS) of 864 RCC patients according to www.proteinatlas.org. (D) ATG12 expression and OS of 864 RCC patients according to www.proteinatlas.org. (E) Immunochemistry analysis of ATG12 expression levels in RCC tissues (T) and normal tissues (N) (×200). RCC, renal cell carcinoma; OS, overall survival.

\section{Conclusions}

In this article, we identified miR $-30 a-3 p$ as a new microRNA that plays a biological function in the invasion and metastasis of RCCs and found its downstream target gene, ATG12, which is a member of the FOXO signaling pathway.

\section{Acknowledgments}

Funding: This study was supported by Award Number 16CR3062B from the Three-Year-Action-Project of Shanghai Hospital Development Centre, Award Number YG2017QN46 from the Medical-Engineering cross fund of Shanghai Jiao Tong University and Award Number 
CBXJ201804 from the Doctoral Innovation Fund of Shanghai Jiao Tong University School of Medicine.

\section{Footnote}

Conflicts of Interest: All authors have completed the ICMJE uniform disclosure form (available at http://dx.doi. org/10.21037/tau.2019.12.10). The authors have no conflicts of interest to declare.

Ethical Statement: The authors are accountable for all aspects of the work in ensuring that questions related to the accuracy or integrity of any part of the work are appropriately investigated and resolved. The use of human tissues was authorized by research medical ethics committee of Shanghai Renji Hospital \{Ethics board approval number: Renji Ethics [2017]091\}.

Open Access Statement: This is an Open Access article distributed in accordance with the Creative Commons Attribution-NonCommercial-NoDerivs 4.0 International License (CC BY-NC-ND 4.0), which permits the noncommercial replication and distribution of the article with the strict proviso that no changes or edits are made and the original work is properly cited (including links to both the formal publication through the relevant DOI and the license). See: https://creativecommons.org/licenses/by-ncnd/4.0/.

\section{References}

1. Bray F, Ferlay J, Soerjomataram I, et al. Global cancer statistics 2018: GLOBOCAN estimates of incidence and mortality worldwide for 36 cancers in 185 countries. CA Cancer J Clin 2018;68:394-424.

2. Torre LA, Bray F, Siegel RL, et al. Global cancer statistics, 2012. CA Cancer J Clin 2015;65:87-108.

3. Chen C, Xue S, Zhang J, et al. DNA-methylationmediated repression of miR-766-3p promotes cell proliferation via targeting SF2 expression in renal cell carcinoma. Int J Cancer 2017;141:1867-78.

4. Ljungberg B, Bensalah K, Canfield S, et al. EAU guidelines on renal cell carcinoma: 2014 update. Eur Urol 2015;67:913-24.

5. Hammond SM. An overview of microRNAs. Adv Drug Deliv Rev 2015;87:3-14.
6. Tan W, Liu B, Qu S, et al. MicroRNAs and cancer: Key paradigms in molecular therapy. Oncol Lett 2018;15:2735-42.

7. Zhai W, Li S, Zhang J, et al. Sunitinib-suppressed miR$452-5 \mathrm{p}$ facilitates renal cancer cell invasion and metastasis through modulating SMAD4/SMAD7 signals. Mol Cancer 2018;17:157.

8. Zhai W, Ma J, Zhu R, et al. MiR-532-5p suppresses renal cancer cell proliferation by disrupting the ETS1-mediated positive feedback loop with the KRAS-NAP1L1/P-ERK axis. Br J Cancer 2018;119:591-604.

9. Turashvili G, Lightbody ED, Tyryshkin K, et al. Novel prognostic and predictive microRNA targets for triplenegative breast cancer. FASEB J 2018:fj201800120R.

10. Wang W, Lin H, Zhou L, et al. MicroRNA-30a-3p inhibits tumor proliferation, invasiveness and metastasis and is downregulated in hepatocellular carcinoma. Eur J Surg Oncol 2014;40:1586-94.

11. Murrow L, Debnath J. ATG12-ATG3 connects basal autophagy and late endosome function. Autophagy 2015;11:961-2.

12. Hu JL, He GY, Lan XL, et al. Inhibition of ATG12mediated autophagy by miR-214 enhances radiosensitivity in colorectal cancer. Oncogenesis 2018;7:16.

13. Yu N, Yong S, Kim HK, et al. Identification of tumor suppressor miRNAs by integrative miRNA and mRNA sequencing of matched tumor-normal samples in lung adenocarcinoma. Mol Oncol 2019;13:1356-68.

14. Cai J, Guan H, Fang L, et al. MicroRNA-374a activates $\mathrm{Wnt} /$ beta-catenin signaling to promote breast cancer metastasis. J Clin Invest 2013;123:566-79.

15. Sun WL, Wang L, Luo J, et al. Ambra1 modulates the sensitivity of breast cancer cells to epirubicin by regulating autophagy via ATG12. Cancer Sci 2018;109:3129-38.

16. Tan D, Zhou C, Han S, et al. MicroRNA-378 enhances migration and invasion in cervical cancer by directly targeting autophagy-related protein 12. Mol Med Rep 2018;17:6319-26.

Cite this article as: Chen $\mathrm{Y}, \mathrm{Zhou} \mathrm{J}, \mathrm{Wu} \mathrm{X}$, Huang $\mathrm{J}$, Chen W, Liu D, Zhang J, Huang Y, Xue W. miR-30a-3p inhibits renal cancer cell invasion and metastasis through targeting ATG12. Transl Androl Urol 2020;9(2):646-653. doi: 10.21037/ tau.2019.12.10 